\title{
Caracterização do cultivo de trigo na região norte do Estado do Rio Grande do Sul através das estimativas oficiais de área cultivada, produção e rendimento de grãos
}

\author{
Characterization of wheat crop in the north region of Rio Grande do Sul State, Brazil, through official \\ estimations of cropping area, grain production and yield
}

\author{
Amanda Heemann Junges ${ }^{*}$ Denise Cybis Fontana ${ }^{\mathrm{I}}$ Ricardo Wanke de Melo $^{\mathrm{I}}$
}

RESUMO

\begin{abstract}
$O$ objetivo deste trabalho foi caracterizar o cultivo de trigo na região norte do Estado do Rio Grande do Sul. Foram empregados dados oficiais de área cultivada e produção de grãos, de 2000 a 2008, dos municípios que compõem as regionais EMATER/RS Caxias do Sul, Erechim, Passo Fundo, Ijuí e Santa Rosa. Os dados foram provenientes do Levantamento Sistemático da Produção Agrícola (LSPA) $I B G E)$. Considerando-se a área cultivada com cereais de estação fria, o trigo tem sido a principal cultura produtora de grãos estabelecida no período de outono-inverno-primavera. A produção de trigo no Estado concentra-se nas regionais analisadas, responsáveis, na média dos anos, por mais de $80 \%$ da quantidade produzida de grãos. Variações interanuais nos rendimentos regionais foram associadas, especialmente, às variações das condições meteorológicas, dada não existência de tendência tecnológica no período. Maiores rendimentos regionais de grãos de trigo e menores coeficientes de variação foram registrados nas regionais localizadas na porção leste (Caxias do Sul e Erechim). A análise de agrupamentos identificou grupos de municípios similares quanto ao rendimento médio municipal de grãos de trigo: baixos

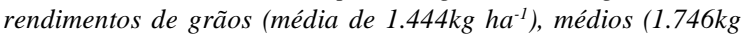
$\left.h a^{-1}\right)$ e altos $\left(2.283 \mathrm{~kg} \mathrm{ha}^{-1}\right)$.
\end{abstract}

Palavras-chave: Triticum aestivum, tendência tecnológica, análise de agrupamentos.

ABSTRACT

This study aimed to characterize the wheat crop in north region of Rio Grande do Sul State, Brazil. It was used data set from official wheat cropping area, grain production and yield, from 2000 to 2008, to the shires that compose the regions of Caxias do Sul, Erechim, Passo Fundo, Ijuí and
Santa Rosa. The data set was obtained from Instituto Brasileiro de Geografia e Estatística (IBGE). Considering winter cereals cropping areas, wheat are the main crop established in the regions during the autumn-winter-spring season. Cropping area estimations indicated that the analyzed regions concentrate the wheat production. The regions produced more than $80 \%$ of state wheat grain production. Since the technological trends weren't registered, the variations in the regional wheat yield were associated with the meteorological conditions during crop cycle. In Rio Grande do Sul State, there was a wheat yield variation in east-west direction: higher yields and low variation coefficients were obtained in the eastern portion (Caxias do Sul and Erechim). The cluster analysis identified tree groups of shires with similar wheat yield: low yield (average $1.444 \mathrm{~kg} \mathrm{ha}^{-1}$ ), medium yield $\left(1.746 \mathrm{~kg} \mathrm{ha}^{-1}\right)$ and high yield $\left(2.283 \mathrm{~kg} \mathrm{ha}^{-1}\right)$.

Key words: Triticum aestivum, technological trends, cluster analysis.

\section{INTRODUÇÃO}

Área cultivada, produção e rendimento de culturas agrícolas são as principais informações que compõem as estimativas de safras. O principal objetivo das estimativas de safras é acompanhar o desenvolvimento dos cultivos de maior importância econômica e social, gerando informações que serão empregadas, por exemplo, na definição de políticas públicas e na formação de preços (ASSAD et al., 2007). No Brasil, estimativas de safras oficiais são de competência do Instituto Brasileiro de Geografia e Estatística (IBGE), responsável por divulgar,

IDepartamento de Plantas Forrageiras e Agrometeorologia, Faculdade de Agronomia, Universidade Federal do Rio Grande do Sul (UFRGS). Av. Bento Gonçalves, 7712, 91540-000, Porto Alegre, RS, Brasil. E-mail: amandahj@hotmail.com. *Autor para correspondência. 
mensalmente, o Levantamento Sistemático da Produção Agrícola (LSPA).

No período de outono-inverno-primavera, no Rio Grande do Sul, as principais culturas produtoras de grãos estabelecidas são trigo, aveia branca, centeio, cevada e triticale. A implantação de lavouras de cereais de estação fria diversifica a produção agrícola, agrega renda ao produtor rural e viabiliza a semeadura direta e a rotação de culturas.

As estimativas de safras indicaram que a produção nacional de trigo oscilou entre 1,7 e 6 milhões de toneladas anuais (IBGE, 2010), de 2000 a 2008. Na média desses anos, a produção gaúcha foi de $1.520 .000 t$, equivalente a $37 \%$ da produção nacional, e os rendimentos de grãos têm oscilado entre $1.355 \mathrm{~kg}$ $\mathrm{ha}^{-1}$ (2006) e 2.266 $\mathrm{kg} \mathrm{ha}^{-1}$ (2008) (IBGE, 2010). De acordo com CANZIANI \& GUIMARÃES (2009), a cultura do trigo apresenta elevada variabilidade de área e rendimento, em função de variações das políticas de exportação e das condições meteorológicas.

As estimativas de safra referentes à cultura do trigo geram informações básicas que podem ser inseridas em estudos de riscos associados à atividade agrícola, modelagem agrometeorológica de rendimento de grãos, programas de melhoramento genético e indicações técnicas de cultivo. Nesse contexto, o objetivo deste trabalho foi caracterizar o cultivo de trigo no Rio Grande do Sul, através dos dados oficiais de área cultivada, produção e rendimento de grãos dos municípios que compõem as regionais Caxias do Sul, Erechim, Passo Fundo, Ijuí e Santa Rosa.

\section{MATERIAL E MÉTODOS}

Foram obtidos dados de área cultivada de aveia branca, centeio, cevada, trigo e triticale (LSPA/ IBGE). Para cultura do trigo, além dos dados de área cultivada, foram analisados dados de área colhida e quantidade produzida de grãos. Os dados de área cultivada referem-se aos disponibilizados pelo LSPA como "área plantada". Os dados foram obtidos, de 2000 a 2008, para o Estado e municípios que compõem as regionais da EMATER/RS Caxias do Sul (58 municípios), Erechim (50), Passo Fundo (70), Ijuí (47) e Santa Rosa (45).

Os dados de área cultivada de aveia branca, centeio, cevada, trigo e triticale foram agrupados de acordo com a regional a que pertence o município. A área cultivada regional correspondeu à soma das áreas cultivadas municipais. Foram somadas as áreas cultivadas com os cereais de estação fria, a fim de que a área cultivada de trigo fosse expressa em termos de porcentagem da área total de cereais de estação fria. Com os dados de área cultivada de trigo foi construído gráfico com a evolução temporal. Foram obtidas as estatísticas descritivas média, desvio padrão e coeficiente de variação (CV) e verificada a diferença entre área cultivada e área colhida de trigo, em cada regional e ano.

O rendimento regional de grãos de trigo foi calculado dividindo-se a quantidade total de grãos produzida na regional pela área colhida total. Foi construído gráfico com a evolução temporal dos rendimentos regionais de grãos de trigo e determinadas as estatísticas descritivas média, desvio-padrão e coeficiente de variação. Para verificação de relação entre os rendimentos regionais de grãos de trigo, foi construída uma matriz de correlação. Duas variáveis são ditas correlacionadas quando mudanças em uma estão associadas a mudanças na outra, ou seja, há uma associação linear entre elas (HAIR JR. et al., 2009). Para construção da matriz de correlação, foram empregados os dados de rendimento regional de grãos de trigo e os rendimentos do Estado, no período. A correlação estabelecida foi a de Pearson, a 1\% de significância estatística.

A existência de tendência tecnológica nos rendimentos regionais de grãos de trigo também foi verificada. Para isso, foi ajustada uma regressão linear simples na qual o rendimento de grãos é a variável dependente e o tempo a variável independente. A significância do coeficiente linear foi verificada pelo teste-t, a 5\% de significância estatística. O termo tendência tecnológica, introduzido em modelagem em 1975 (BRAGA, 1995), refere-se a aumentos anuais significativos no rendimento de grãos decorrentes da incorporação de novas tecnologias.

Semelhante às análises regionais, os rendimentos municipais de grãos de trigo foram obtidos dividindo-se a quantidade produzida municipal pela área colhida municipal. Foram obtidos valores máximo, mínimo e médio para cada regional e ano. Também foi calculado o rendimento de grãos de trigo, de cada um dos municípios, na média dos anos da série. Para os rendimentos médios municipais foram determinados média, mediana, desvio padrão e coeficiente de variação e avaliada a normalidade dos dados pelo teste de Kolmogorov-Smirnov (5\% de significância estatística). A forma de distribuição dos dados pode ser semelhante à normal. A distribuição normal é padrão e serve de referência para uma série de métodos estatísticos (HAIR JR. et al., 2009). O teste de Kolmogorov-Smirnov calcula o nível de significância das diferenças em relação a uma distribuição normal (HAIR JR.et al., 2009).

Com os rendimentos municipais de grãos de trigo de 2001 a 2008 foi realizada uma análise de agrupamentos. A análise de agrupamentos é um conjunto de técnicas multivariadas cuja finalidade principal é agregar objetos com base nas características que estes possuem (HAIR JR. et al., 2009). Neste trabalho, a análise de agrupamentos foi realizada pelo 
método de Ward, que utiliza a distância euclidiana ao quadrado como medida da similaridade entre objetos. Foram gerados três grupos de municípios e calculados os rendimentos médios. O resultado foi espacializado com uso do programa Carta Linx.

\section{RESULTADOS E DISCUSSÃO}

A evolução temporal da área cultivada de trigo (Figura 1) indicou que no Estado, de 2000 a 2004, a área cultivada aumentou, ultrapassando um milhão

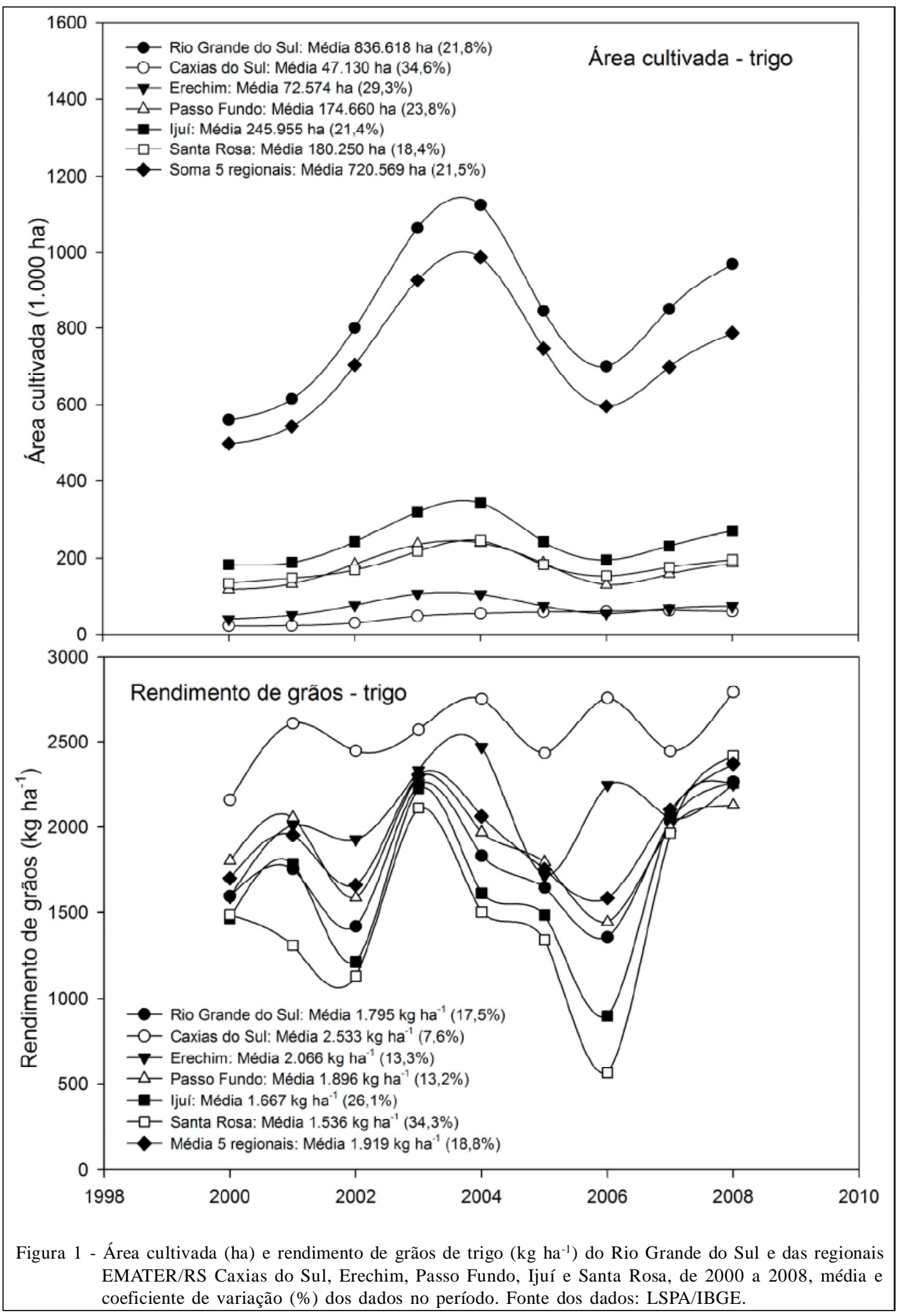

Ciência Rural, v.42, n.1, jan, 2012. 
de hectares. Em 2005 e 2006, foi registrada diminuição de área (699.451ha), com novo incremento em 2007 (850.169ha)e 2008 (970.375ha). Aárea cultivada média, noEstado, foi de 836.618ha, com coeficiente de variação de $22 \%$ e diferença de 565.000 ha entre os valores máximo (1.125.000ha, ano 2004) e mínimo (560.560ha, ano 2000). A cultura do trigo caracteriza-se pela elevada variabilidade de área e de rendimento, em virtude das condições meteorológicas e das políticas econômicas do País. Na questão econômica, de 1999 a 2004, a área cultivada de trigo aumentou, dado os maiores preços do produto no mercado interno. De 2005 a 2007, as importações se tornaram a principal fonte de trigo. Aárea cultivada de trigo voltou a crescer em 2008 , em virtude do alto preço das commodities agrícolas no mercado internacional (CANZIANI \& GUIMARÃES, 2009).

A área cultivada de trigo na soma das cinco regionais foi equivalente a $86 \%$ da área cultivada no Estado, na média dos anos. A partir desse resultado, foi possível indicar que a produção gaúcha de trigo concentrou-se nas regionais analisadas. Em todos os anos, a maior área cultivada de trigo ocorreu na regional Ijuí (entre 27 e $33 \%$ da área cultivada no Estado), seguida por Santa Rosa (entre 20 e 24\%) e Passo Fundo (entre 19 e 23\%). Os maiores coeficientes de variação de área cultivada foram registrados em Caxias do Sul (37\%) e Erechim (31\%) e o menor em Santa Rosa (19\%). As regionais Passo Fundo e Ijuí apresentaram coeficientes de variação da área cultivada de trigo de $25 \%$ e $23 \%$, respectivamente.

Considerando-se a área total cultivada com cereais de estação fria, o trigo foi a cultura que ocupou a maior área, em todas as regionais: Caxias do Sul (53 a 85\%), Erechim (57 a 87\%), Passo Fundo (61 a 85\%), Ijuí (84 a 94\%) e Santa Rosa (96 a 98\%). Assim, o trigo tem sido a principal cultura produtora de grãos estabelecida no período de outono-inverno-primavera.

A evolução temporal da área colhida de trigo no Estado, nas regionais e na soma das cinco regionais foi semelhante à evolução da área cultivada. A área colhida nas regionais correspondeu a $86 \%$ da área colhida no Estado, na média dos anos (variação de
$81 \%$ a 89\%). A diferença entre área cultivada e área colhida é uma informação simples que, associada ao calendário agrícola e às informações meteorológicas, auxilia na caracterização das quebras de safras. Sendo assim, foi possível verificar que a maior diferença ocorreu em 2006, ano em que 92.182ha deixaram de ser colhidos no Estado. Aproximadamente 97\% desta área corresponderam à soma da área não colhida nas regionais analisadas. As maiores áreas deixaram de ser colhidas em Ijuí (47.530ha) e Santa Rosa (39.000ha). As geadas, ocorridas em agosto e setembro, foram responsáveis pela quebra de safra ocorrida em 2006, quando 50\% da área cultivada de trigo no Estado foi atingida por este evento meteorológico (JUNGES et al., 2008). Nos demais anos, a diferença entre as áreas situou-se entre 100ha (2008) e 6.120ha (2000).

A evolução temporal dos rendimentos regionais de grãos de trigo (Figura 1) mostrou que as regionais Caxias do Sul, Erechim e Passo Fundo obtiveram rendimentos superiores à média estadual $\left(1795 \mathrm{~kg} \mathrm{ha}^{-1}\right)$, na maioria dos anos. Os maiores rendimentos médios regionais ocorreram em Caxias do Sul $\left(2.553 \mathrm{~kg} \mathrm{ha}^{-1}\right)$, Erechim (2.066 $\left.\mathrm{kg} \mathrm{ha}^{-1}\right)$ e Passo Fundo $\left(1.896 \mathrm{~kg} \mathrm{ha}^{-1}\right)$. Ijuí e Santa Rosa caracterizaram-se por menores valores, respectivamente $1.667 \mathrm{~kg} \mathrm{ha}^{-1} \mathrm{e}$ $1.536 \mathrm{~kg} \mathrm{ha}^{-1}$.

Os rendimentos regionais apontaram para existência de variação espacial, no sentido leste-oeste, do rendimento de grãos de trigo no Estado. Os maiores rendimentos foram registrados nas regionais localizadas na porção leste da área de estudo. Por sua vez, em termos de coeficiente de variação dos rendimentos regionais de grãos de trigo, foi verificada ordem inversa. De acordo com CUNHA et al. (2009), regiões de maior altitude têm sido as que apresentam maior rendimento de grãos e menor variabilidade entre safras, especialmente em função da relação entre radiação solar e temperatura do ar (quociente fototermal) no período de 30 dias próximos à antese. $\mathrm{O}$ decréscimo do quociente fototermal no sentido lesteoeste faz com que os rendimentos de grãos também diminuam neste sentido (CUNHA et al., 2005).

A matriz de correlação (Tabela 1) indicou que, no Estado, dois grupos de regionais podem ser

Tabela 1 - Matriz de correlação entre os rendimentos de grãos de trigo (2000 a 2008) do Rio Grande do Sul (RS), das regionais EMATER/RS Caxias do Sul, Erechim, Passo Fundo, Ijuí e Santa Rosa e rendimento médio das cinco regionais. Fonte dos dados: LSPA/IBGE.

\begin{tabular}{lllccccc}
\hline & RS & Caxias & Erechim & Passo Fundo & Ijuí & Santa Rosa & Médio \\
\hline Rio Grande do Sul & 1,00 & & & & & & \\
Caxias & 0,27 & 1,00 & & & & & \\
Erechim & 0,44 & $0,86^{*}$ & 1,00 & & & & \\
Passo Fundo & $0,93^{*}$ & 0,15 & 0,34 & 1,00 & & & \\
Ijuí & $0,97^{*}$ & 0,13 & 0,29 & $0,95^{*}$ & 1,00 & & 1,00 \\
Santa Rosa & $0,95^{*}$ & 0,05 & 0,22 & $0,86^{*}$ & $0,95^{*}$ & $0,92^{*}$ & 1,00 \\
Médio & $0,99^{*}$ & 0,38 & 0,55 & $0,92^{*}$ & $0,95^{*}$ & & \\
\hline
\end{tabular}

* Correlação de Pearson, a 1\% de significância estatística 
relacionados (linearmente) em termos de rendimento de grãos de trigo: um grupo foi formado pelas regionais Caxias do Sul e Erechim e outro pelas regionais Passo Fundo, Ijuí e Santa Rosa. O rendimento estadual de trigo foi significativamente relacionado ao rendimento de Passo Fundo (0,93), Ijuí $(0,97)$ e Santa Rosa $(0,95)$, bem como ao rendimento médio das cinco regionais $(0,99)$. A identificação da associação entre o rendimento obtido por diferentes regionais contribui para melhor compreensão do efeito de características edafo-climáticas no crescimento e desenvolvimento das plantas de trigo em lavouras situadas na porção leste (Caxias e Erechim) e porção central-oeste (Passo Fundo, Ijuí e Santa Rosa) da região produtora de trigo no Estado.
No que diz respeito à tendência tecnológica, os resultados da regressão linear indicaram que não houve incremento significativo no rendimento de grãos de trigo das regionais, no período analisado. CUNHA et al. (1999) verificaram a existência de tendência tecnológica no rendimento de grãos de trigo do Estado, empregando uma série maior de dados (1920 a 1997). Dada ausência de tendência tecnológica no período analisado neste trabalho, as variações interanuais do rendimento de grãos puderam ser atribuídas às variações das condições meteorológicas.

Os valores máximo, mínimo e médio dos rendimentos municipais constam na tabela 2 . Considerando-se os valores máximos de rendimentos

Tabela 2 - Valor máximo, mínimo e médio dos rendimentos municipais de grãos trigo (kg ha ${ }^{-1}$ ) (A), estatísticas descritivas (média, mediana, desvio padrão, coeficiente de variação) e teste de normalidade dos dados (Kolmogorov-Smirnov) dos rendimentos médios municipais de grãos de trigo $\left(\mathrm{kg} \mathrm{ha}^{-1}\right)(\mathrm{B})$, regionais EMATER/RS Caxias do Sul, Erechim, Passo Fundo, Ijuí e Santa Rosa, 2000 a 2008. Fonte dos dados: LSPA/IBGE.

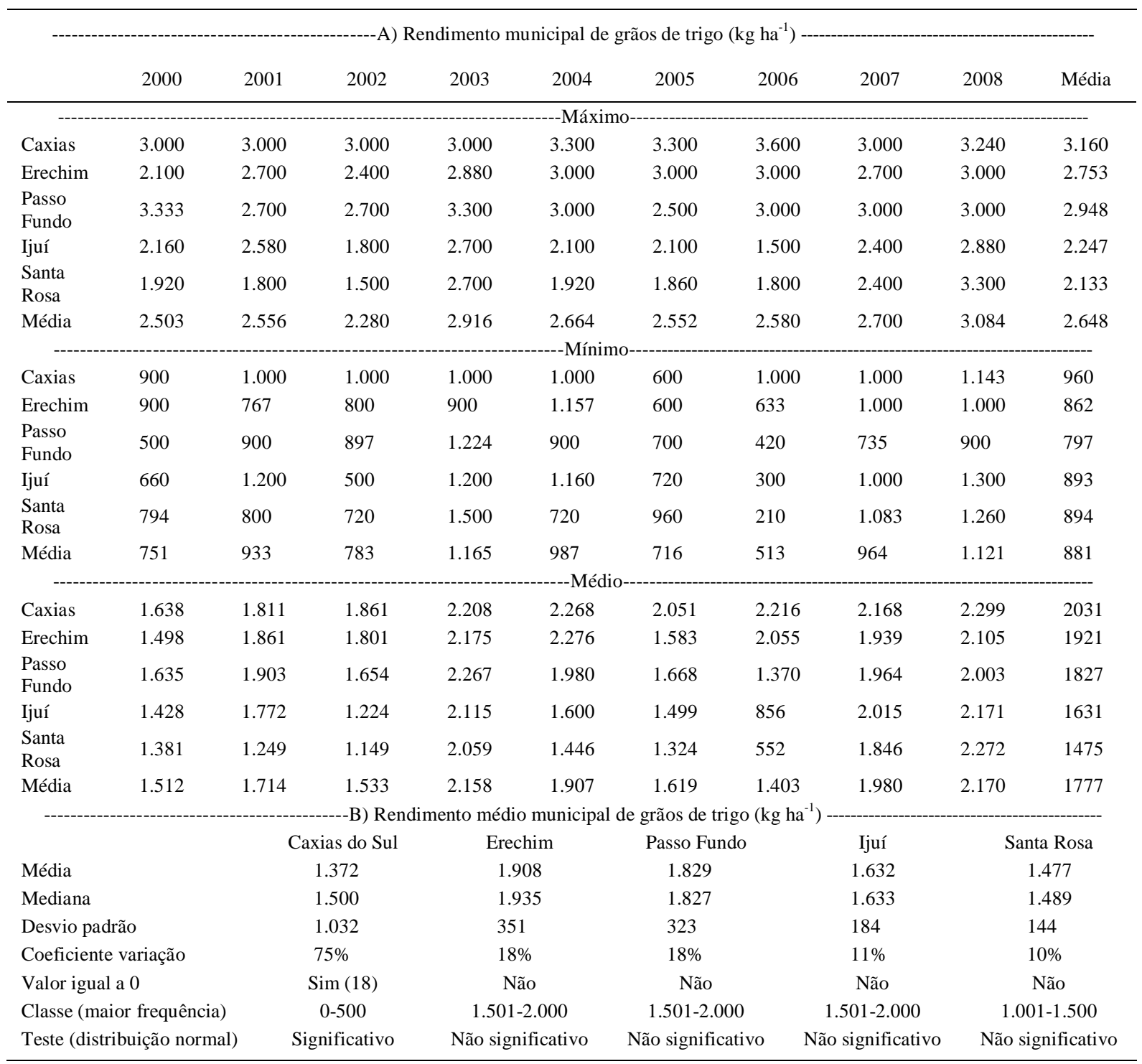

Ciência Rural, v.42, n.1, jan, 2012. 
municipais de grãos de trigo, a maior média foi registrada na regional Caxias do Sul $\left(3.160 \mathrm{~kg} \mathrm{ha}^{-1}\right)$, seguida por Passo Fundo (2.948 $\left.\mathrm{kg} \mathrm{ha}^{-1}\right)$ e Erechim (2.753 $\left.\mathrm{kg} \mathrm{ha}^{-1}\right)$. O maior valor absoluto $\left(3.600 \mathrm{~kg} \mathrm{ha}^{-1}\right)$ também foi registrado em município pertencente à regional Caxias doSul (Guabiju, em 2006). A regional Santa Rosa, apesar de possuir, na média dos anos, rendimento máximo inferior ao das demais $\left(2.133 \mathrm{~kg} \mathrm{ha}^{-1}\right)$, registrou incremento em 2007 e 2008. Considerando-se os valores mínimos de rendimentos municipais de grãos de trigo, a menor média ocorreu na regional Passo Fundo $(797 \mathrm{~kg}$ $\mathrm{ha}^{-1}$ ) e o menor valor absoluto foi $210 \mathrm{~kg} \mathrm{ha}^{-1}$, em Tucunduva (regional Santa Rosa), em 2006. As maiores amplitudes nos rendimentos municipais de grãos de trigo ocorreram em anos distintos: nas regionais Caxias do Sul e Erechim (2.700 e 2.400 $\mathrm{kg} \mathrm{ha}^{-1}$ ) em 2005, Passo Fundo (2.833 $\left.\mathrm{kg} \mathrm{ha}^{-1}\right)$ em 2000 e Ijuí e Santa Rosa (1.580 e $\left.2.040 \mathrm{~kg} \mathrm{ha}^{-1}\right) \mathrm{em} 2008$. As maiores amplitudes médias ocorreram nas regionais Caxias do Sul $\left(2.200 \mathrm{~kg} \mathrm{ha}^{-1}\right) \mathrm{e}$ Passo Fundo (2.151 kg ha- ${ }^{-1}$.

Considerando-se os rendimentos médios municipais, os valores máximos foram obtidos pelos municípios Capão Bonito do Sul (regional Caxias do Sul, 2.913 $\mathrm{kg} \mathrm{ha}^{-1}$ ), Sananduva (regional Erechim, $2.507 \mathrm{~kg}$ $\mathrm{ha}^{-1}$ ), Água Santa (regional Passo Fundo, 2.604kg ha ${ }^{-1}$ ), Sede Nova (regional Ijuí, $1.940 \mathrm{~kg} \mathrm{ha}^{-1}$ ) e Rolador (regional Santa Rosa, $1.808 \mathrm{~kg} \mathrm{ha}^{-1}$ ). O teste de Kolmogorov-Smirnov indicou a não normalidade dos dados de rendimento médio municipal na regional Caxias do Sul (Tabela 2). Além disso, na regional Caxias do Sul, a média dos rendimentos médios municipais $\left(1.372 \mathrm{~kg} \mathrm{ha}^{-1}\right)$ foi inferior à mediana $\left(1.500 \mathrm{~kg} \mathrm{ha}^{-1}\right)$ e não se situou na classe de maior frequência $\left(0 \mathrm{a} 500 \mathrm{~kg} \mathrm{ha}^{-1}\right)$ (Tabela 2). O coeficiente de variação dos dados foi elevado (75\%). Esses resultados foram decorrentes da existência, no conjunto de dados, de 18 municípios com rendimentos médios iguais a zero. Nas demais regionais, os dados apresentaram distribuição normal, a média foi próxima da mediana e localizada na classe de maior frequência, logo, representativa dos rendimentos médios municipais de grãos de trigo. $\mathrm{O}$ teste de Kolmogorov-Smirnov confirmou a distribuição normal dos dados nas regionais Erechim, Passo Fundo, Ijuí e Santa Rosa.

$\mathrm{Na}$ análise de agrupamentos foram formados três grupos similares quanto ao rendimento municipal de grãos de trigo de 2001 a 2008 (Figura 2). Orendimento médio dos grupos foi de $1.444 \mathrm{~kg} \mathrm{ha}^{-1}$ (grupo 1), $1.746 \mathrm{~kg}$

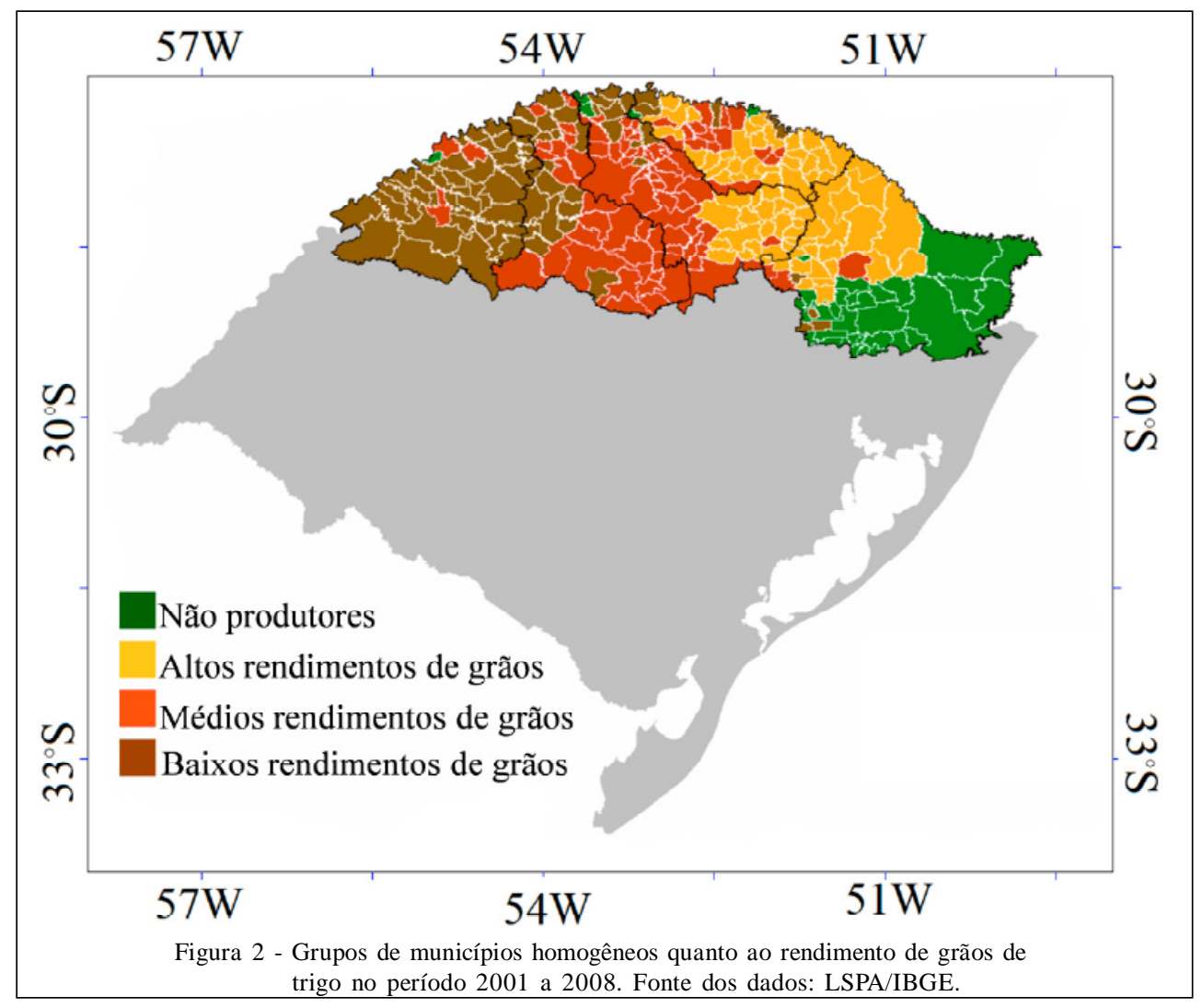

Ciência Rural, v.42, n.41, jan, 2012. 
$\mathrm{ha}^{-1}$ (grupo 2) e $2.283 \mathrm{~kg} \mathrm{ha}^{-1}$ (grupo 3), sendo denominados, respectivamente, de grupo de baixos, médios e altos rendimentos de grãos de trigo. No grupo de baixos rendimentos foram inseridos 76 municípios, no de médios rendimentos, 88 municípios e, no de altos rendimentos, 71. Trinta e cinco municípios não foram inseridos nos grupos formados, resultado decorrente da falta do dado em, pelo menos, um dos anos da série. Desses 35 municípios, 30 estão localizados na regional Caxias do Sul e, destes, 18 não produziram trigo em nenhum dos anos analisados.

A análise de agrupamentos, bem como a espacialização desta, identificou as diferenças em termos de rendimento de grãos de trigo, entre regionais e, principalmente, entre municípios que compõem uma mesma regional. Na regional Caxias do Sul, apesar da existência do maior número de municípios não produtores, os produtores estão inseridos no grupo altos rendimentos. Os municípios da regional Erechim foram inseridos nos grupos de alto e médio rendimento. Como não produtores de trigo foram identificados os municípios Ametista do Sul e Mariano Moro. Os municípios da regional Passo Fundo foram inseridos nos três grupos: aqueles localizados próximos à regional Caxias do Sul pertenceram ao grupo de alto rendimento, os localizados na porção central da regional pertenceram ao grupo de médio rendimento, e os municípios localizados próximos à regional Ijuí foram inseridos no grupo de baixo rendimento. Palmitinho e Pinheirinho do Vale foram considerados municípios não produtores. Nas regionais Ijuí e Santa Rosa, a maioria dos municípios pertenceu ao grupo de baixos rendimentos. Na regional Ijuí, todos os municípios foram considerados produtores. Na regional Santa Rosa, Porto Vera Cruz foi o município não produtor de trigo, no período analisado.

A análise de agrupamentos foi considerada uma técnica exploratória eficiente na detecção de municípios semelhantes, no que diz respeito à evolução temporal do rendimento municipal de grãos de trigo. Esta análise evidenciou a existência de gradiente lesteoeste nos rendimentos de grãos de trigo, concordando a variação do quociente fototermal no Estado. Diferenças de rendimento de grãos de trigo existentes entre municípios vizinhos podem ser associadas às características edafo-climáticas, sistemas de manejo, cultivares e demais fatores determinantes da produção de grãos. Os resultados deste trabalho sintetizaram as informações contidas nas estimativas oficiais de safras e permitiram a caracterização do cultivo de trigo no Estado.

\section{CONCLUSÃO}

A análise das estimativas oficiais de safra de trigo dos municípios pertencentes às regionais EMATER/RS Caxias do Sul, Erechim, Passo Fundo, Ijuí e Santa Rosa permitiram concluir que a produção gaúcha de trigo concentra-se na metade norte do Estado. Tendo por base a área cultivada, o trigo é a principal cultura produtora de grãos estabelecida no período de outono-inverno-primavera. Há variação dos rendimentos regionais de grãos de trigo, no sentido leste-oeste: maiores rendimentos e menor variação entre safras ocorrem na porção leste. As variações interanuais dos rendimentos regionais de grãos de trigo estão associadas às condições meteorológicas ocorridas ao longo do ciclo, dada não existência de tendência tecnológica no período. Quanto ao rendimento municipal de grãos de trigo, três grupos homogêneos podem ser formados (altos, médios e baixos rendimentos). A localização espacial desses grupos ressalta existência de gradiente leste-oeste nos rendimentos de grãos de trigo no Estado.

\section{REFERÊNCIAS}

ASSAD, E.D. et al. Sistema de previsão da safra de soja para o Brasil. Pesquisa Agropecuária Brasileira, v.42, n.5, p.615625, 2007. Disponível em: <http://www.scielo.br/pdf/pab/v42n5/ 02.pdf>. Acesso em: 07 set. 2011.

BRAGA, H.J. Previsão agrícola: uma nova abordagemuso de scanner aerotransportável e redes neurais. 1995. 197f. Tese (Doutorado em Engenharia de Produção) Universidade Federal de Santa Catarina, Florianópolis, SC.

CANZIANI, J.R., GUIMARÃES, V.D.A. O trigo no Brasil e no mundo: cadeia de produção, transformação e industrialização. In: CUNHA, G.R. Oficina sobre trigo no Brasil - Bases para construção de uma nova triticultura brasileira. Passo Fundo: Embrapa Trigo, 2009, p.29-72.

CUNHA, G.R. et al. Enso influences on wheat crop in Brazil. Revista Brasileira de Agrometeorologia, v.7, n.1, p.127$138,1999$.

CUNHA, G.R. et al. Variabilidade temporal e espacial do quociente fototermal no Rio Grande do Sul e suas implicações para a expressão do potencial de rendimento de grãos de trigo. Revista Brasileira de Agrometeorologia, v.13, n.1, p.91$101,2005$.

CUNHA, G.R. et.al. Trigo. In: MONTEIRO, J.E.B.A. (Org.). Agrometeorologia dos cultivos - o fator meteorológico na produção agrícola. Brasília: INMET, 2009. p.281-293.

HAIR JR, F. et al. Análise de regressão múltipla. In: HAIR JR. et al. Análise multivariada de dados. 6.ed. Porto Alegre: BOOKMAN, 2009. p.149-220.

IBGE. Levantamento sistemático da produção agrícola. Disponível em: 〈http://www.sidra.ibge.gov.br/bda/agric〉. Acesso em: 10 jun. 2010

JUNGES, A.H. et al. Avaliação das perdas de rendimento de trigo na safra 2006 por meio das análises de temperaturas mínimas do ar e de superfície na região norte do Rio Grande do Sul. Revista Brasileira de Agrometeorologia, v.16, n.1, p.1-8, 2008. Disponível em: <http://www.sbagro.org.br/rbagro/ ojs/index.php/rbagro/article/view/81/24>. Acesso em: 07 set. 2011. 